\title{
Clinical Use of Next-Generation Sequencing in the Diagnosis of Wilson's Disease
}

\author{
Dániel Németh, ${ }^{1}$ Kristóf Árvai, ${ }^{2}$ Péter Horváth, ${ }^{1}$ János Pál Kósa, ${ }^{1,2}$ \\ Bálint Tobiás, ${ }^{2}$ Bernadett Balla, ${ }^{2}$ Anikó Folhoffer, ${ }^{1}$ Anna Krolopp, ${ }^{1}$ \\ Péter András Lakatos, ${ }^{1}$ and Ferenc Szalay ${ }^{1}$ \\ ${ }^{1} 1$ st Department of Internal Medicine, Semmelweis University, Koranyi Sandor Street 2/a, Budapest 1083, Hungary \\ ${ }^{2}$ PentaCore Lab, Koranyi Sandor Street 2/a, Budapest 1083, Hungary
}

Correspondence should be addressed to Dániel Németh; neemethd@gmail.com

Received 8 July 2015; Revised 15 September 2015; Accepted 20 September 2015

Academic Editor: Alfred Gangl

Copyright (C) 2016 Dániel Németh et al. This is an open access article distributed under the Creative Commons Attribution License, which permits unrestricted use, distribution, and reproduction in any medium, provided the original work is properly cited.

Objective. Wilson's disease is a disorder of copper metabolism which is fatal without treatment. The great number of disease-causing $A T P 7 B$ gene mutations and the variable clinical presentation of WD may cause a real diagnostic challenge. The emergence of nextgeneration sequencing provides a time-saving, cost-effective method for full sequencing of the whole ATP7B gene compared to the traditional Sanger sequencing. This is the first report on the clinical use of NGS to examine ATP7B gene. Materials and Methods. We used Ion Torrent Personal Genome Machine in four heterozygous patients for the identification of the other mutations and also in two patients with no known mutation. One patient with acute on chronic liver failure was a candidate for acute liver transplantation. The results were validated by Sanger sequencing. Results. In each case, the diagnosis of Wilson's disease was confirmed by identifying the mutations in both alleles within 48 hours. One novel mutation (p.Ala1270Ile) was found beyond the eight other known ones. The rapid detection of the mutations made possible the prompt diagnosis of WD in a patient with acute liver failure. Conclusions. According to our results we found next-generation sequencing a very useful, reliable, time-saving, and cost-effective method for diagnosing Wilson's disease in selected cases.

\section{Introduction}

Wilson's disease (WD) is a rare autosomal recessive disorder of copper metabolism. ATP7B gene mutation is in the background of the excessive copper accumulation which is fatal without treatment. More than 550 disease-causing mutations of the gene located at chromosome 13q14.3-q21.1 consisting of 21 exons have been identified [1].

The geographical distribution of the mutations of the $A T P 7 B$ gene is inhomogeneous [2-5]. In Hungary the p.His1069Gln mutation is the most frequent one with $71 \%$ prevalence among the patients [6].

The variable clinical presentation of WD may cause a real diagnostic challenge. The suspicion of the disease usually arises when hepatic or neurologic-psychiatric symptoms appear. Low ceruloplasmin level and presence of KayserFleischer ring could support the diagnosis, but in many cases only genetic testing could confirm it. Genetic investigation of asymptomatic siblings has an extreme importance, since the early treatment could prevent the manifestation of the disease [7]. In acute liver failure urgent genetic testing of all known mutations may strengthen the diagnosis of Wilson's disease.

The emergence of next-generation sequencing (NGS) provides a time-saving, cost-effective method for full sequencing of the whole $A T P 7 B$ coding sequence compared to the traditional Sanger sequencing. The NGS technology is based on the detection of a signal during the synthesis of the DNA strand, and therefore the synthesis does not need to be terminated for the perception. On the other hand, several DNA strands can be examined simultaneously [8].

This is the first report on the clinical use of NGS to examine $A T P 7 B$ gene in WD patients including doubtful cases. We used Ion Torrent Personal Genome Machine in heterozygous patients for the identification of the other 
mutations and also in patients with no known mutation including one with acute on chronic liver failure.

\section{Materials and Methods}

The method we used for the genetic testing has been previously published by our group for screening of neurofibromatosis type 1 gene [9].

2.1. Biological Samples and DNA Isolation. Six (five male and one female) WD patients, four heterozygous for $A T P 7 B$ p.His1069Gln mutation identified by fast PCR test and two with unknown mutation, were selected for this study. The patients were diagnosed and treated at the 1st Department of Internal Medicine, Semmelweis University, Budapest. The diagnosis was based on the international WD score system published in 2003 [10], and each patient had 4 or more scores. The study was approved by the Semmelweis University's Committee of Research Ethics and was conducted in accordance with the Helsinki Declaration. All patients gave written informed consent.

Genomic DNA was isolated from $200 \mu \mathrm{L}$ of peripheral blood using ReliaPrep Blood gDNA Miniprep System (Promega, Madison, WI). Briefly, the blood samples were digested with Proteinase $\mathrm{K}$ solution in the presence of Cell Lysis Buffer, and, after $10 \mathrm{~min}$ of incubation at $56^{\circ} \mathrm{C}$, DNA was bound to ReliaPrep Binding Column. After three washes, DNA was eluted into $50 \mu \mathrm{L}$ of nuclease-free water. The concentration of the isolated DNA was determined with Qubit dsDNA HS Assay Kit (Life Technologies, Carlsbad, CA).

2.2. Ion Torrent Sequencing. ATP7B (21 coding exons) amplicons were designed using the AmpliSeq Designer software (Life Technologies, CA, USA), targeting the complete coding sequence of $A T P 7 B$ gene, resulting in a total of 55 amplicons. To gain a higher coverage of the coding exons, we designed the primers to also flank some parts of the introns. Amplicon library was prepared using the Ion AmpliSeq Library Kit 2.0 (Life Technologies, CA, USA); briefly, multiplex primer pools were added to $10 \mathrm{ng}$ of genomic DNA and amplified with the following PCR cycles: at $99^{\circ} \mathrm{C}$ for $2 \mathrm{~min}$, at $99^{\circ} \mathrm{C}$ for $15 \mathrm{~s}$, and at $60^{\circ} \mathrm{C}$ for $4 \mathrm{~min}$ (18 cycles), and holding on at $10^{\circ} \mathrm{C}$. Primers were partially digested using a FuPa reagent, and then sequencing adapters were ligated to the amplicons. The library was purified in multiple times using the Agencourt AMPure XP Reagent (Beckmann Coulter, CA, USA). The concentration of the final library was determined by fluorescent measurement on Qubit 2.0 instrument (Life Technologies, CA, USA). Template preparation was performed with Ion OneTouch kit (Life Technologies, CA, USA) on semiautomatic Ion OneTouch instrument using an emPCR method. After breaking the emulsion, the nontemplated beads were removed from the solution during the semiautomatic enrichment process on Ion OneTouch ES (Life Technologies, CA, USA) instrument. After adding the sequencing primer and polymerase, the fully prepared Ion Sphere Particle (ISP) beads were loaded into an Ion 314 v2 sequencing chip, and the sequencing runs were performed using the Ion PGM 200 Sequencing kit v2 (Life Technologies, CA, USA) with 500 flows.

2.3. Sanger Sequencing Validation. The PCR primers were designed using Primer3Plus (http://primer3plus.com/) software. Roche FastStart TaqMan Probe Master (Roche) kit was used to amplify the target regions and the PCR program was as follows: $95^{\circ} \mathrm{C}$ for $10 \mathrm{~min}, 40$ cycles of $95^{\circ} \mathrm{C}$ for $30 \mathrm{~s}$, $60^{\circ} \mathrm{C}$ for $30 \mathrm{~s}$, and $72^{\circ} \mathrm{C}$ for $45 \mathrm{~s}$, and the final step was $72^{\circ} \mathrm{C}$ for $5 \mathrm{~min}$. PCR products were enzymatically cleaned using ExoSAP IT (Affymetrix, Santa Clara, CA) according to the manufacturer's instructions. Sanger sequencing was performed using BigDye Terminator v3.1 Cycle Sequencing Kit (Life Technologies) using an ABI 3130 instrument (Life Technologies).

2.4. Data Analysis. Data from the Ion Torrent runs were analyzed using the platform-specific pipeline software Torrent Suite v3.6 for base calling, trim adapter and primer sequences, filter out poor quality reads, and demultiplex the reads according to the barcode sequences. Briefly, TMAP (https:/github.com/iontorrent/TMAP) algorithm was used to align the reads to the hg19 human reference genome, and then the variant caller plug-in was selected to run to search for germ line variants in the targeted regions. The variant caller algorithm parameters were more relaxed to avoid false negative cases. Integrative Genomics Viewer was used for visualization of the mapped reads. Variants were reviewed and annotated using dsSNP (http://www.ncbi.nlm.nih.gov /projects/SNP/) and Wilson Disease Mutation Database (http://www.wilsondisease.med.ualberta.ca/index.asp). For variant interpretation, Ingenuity Variant Analysis Pipeline (Ingenuity, Rewood City, CA) was also used. Pathogenic status of the variant was stated if it was a missense variant with $<1 \%$ minor allele frequency and/or the variant was listed in the literature or in the databases as a pathogenic alteration. All of the deleterious variants were confirmed by Sanger sequencing. The Sanger sequence data were investigated using ABI Sequence Scanner 1.0 (Life Technologies) and BioEdit (http://www.mbio.ncsu.edu/bioedit/bioedit.html) software.

\section{Results}

The demographic and clinical characteristics of the patients are shown in Table 1. One patient without known mutation was critically ill with acute on chronic liver disease. The typical laboratory findings (ALT 90, AST 178, ALP 88, and bilirubin 247) proposed Wilson's disease. The diagnosis was strengthened by genetic testing making possible the liver transplantation via Eurotransplant program in Patient 5 with acute on chronic liver failure.

In each case, the diagnosis of Wilson's disease was confirmed by identifying the mutations in both alleles. The results were available within 48 hours.

The average read number per sample was 134386 , with an average $1 \mathrm{X}$ on-target coverage of $99.46 \%$. The mean raw 
TABLE 1: Demographic and clinical characteristics of the patients.

\begin{tabular}{|c|c|c|c|c|c|c|c|c|c|c|c|}
\hline & Gender & $\begin{array}{c}\text { Age at onset } \\
\text { (year) }\end{array}$ & KFR & Neu & $\mathrm{HA}$ & Urin Cu & Biopsy & Cerul (g/L) & $A T P 7 B$ status & WD score ${ }^{a}$ & Phenotype \\
\hline Patient 1 & Female & 12 & $\mathrm{P}$ & A & A & ++ & ND & 0.18 & p.Met769-fs/p.His1069Gln & 6 & $S$ \\
\hline Patient 2 & 2 Male & 17 & A & $\mathrm{P}$ & A & + & ND & 0.05 & p.Ala1063Val/p.His1069Gln & 6 & N1 \\
\hline Patient 3 & Male & 8 & $\mathrm{P}$ & A & A & ++ & $+^{\mathrm{b}}$ & 0.06 & p.His1069Gln/p.Gln1351Stop & 8 & $\mathrm{H} 2$ \\
\hline Patient 4 & 4 Male & 17 & $\mathrm{P}$ & A & A & + & ND & 0.03 & p.Ala1135-fs/p.Leu1305Pro & 5 & $\mathrm{H} 2$ \\
\hline Patient 5 & 5ale & 44 & A & A & A & ++ & ND & 0.08 & p.Ala1270Ile/c.1707+2dupT & 4 & $\mathrm{H} 1$ \\
\hline Patient 6 & 5 Male & 14 & $\mathrm{P}$ & $\mathrm{P}$ & A & ND & ND & 0.04 & p.Arg969Gln/p.His1069Gln & 7 & $\mathrm{~N} 2$ \\
\hline
\end{tabular}

KFR: Kayser-Fleischer ring; Neu: neurological signs and/or CT/MRI alterations; HA: hemolytic anemia; Urin Cu: urinary copper, 1-2X ULN: +, >2x ULN or positive D-penicillamine challenge: ++; Cerul: ceruloplasmin, P: present; A: absent; ND: not done; S: sibling; H1: acute liver failure; H2: chronic liver disease; N1: neurological symptoms with liver disease; N2: only neurological symptoms.

${ }^{a}$ According to the international score system, 4 or more scores, diagnosis of WD is highly likely. ${ }^{b}$ Rhodanine positivity.

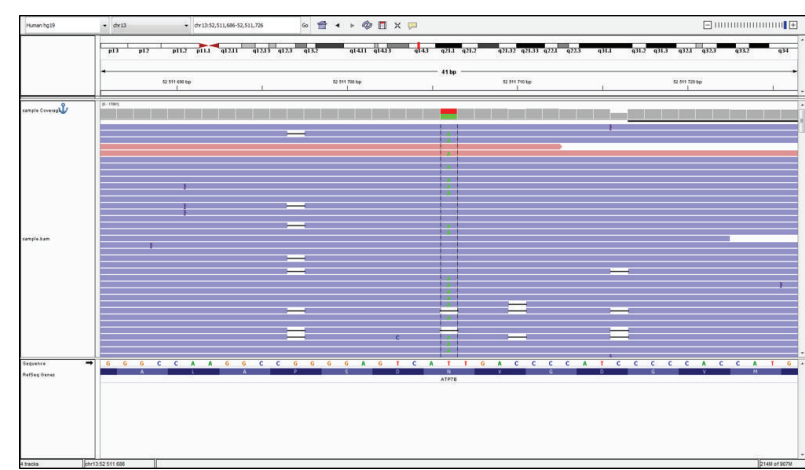

(a1)

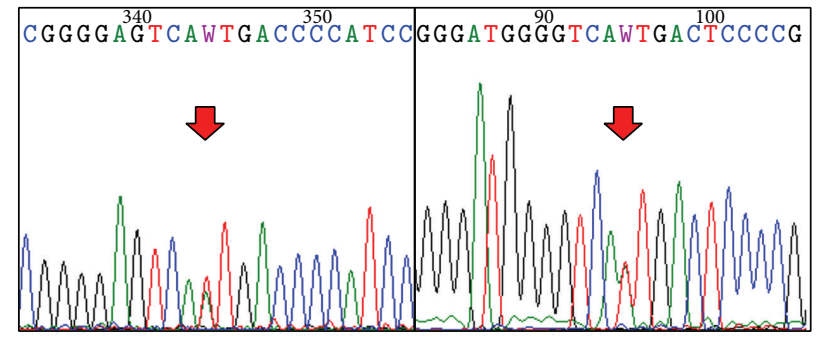

(b1)

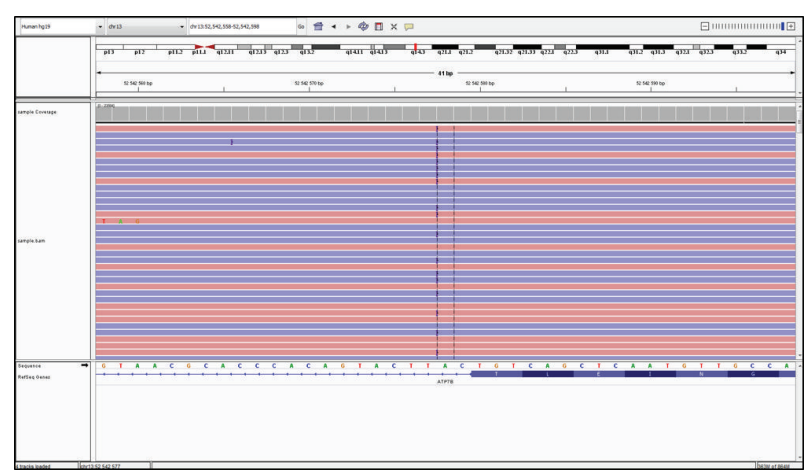

(a2)

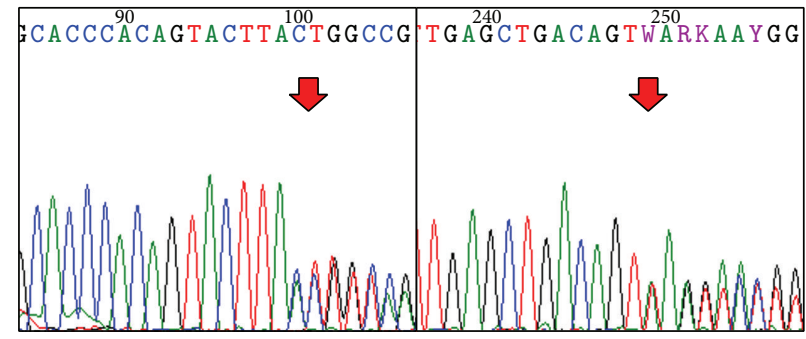

(b2)

FIGURE 1: The identified mutations of patient 5. Both c.3809A $>$ T (causing amino acid change p.Ala1270Ile) and c.1707+2dupT mutations are confirmed by Sanger-sequencing. The 3809A $>C$ and $A>G$ mutations are known, but the $A>T$ substitution is a novel alteration at this position. (al) Visualizing the alignment of the sequencing reads covering the $A T P 7 B$ c.3809A $>T$ heterozygous point mutation. The coverage was 400-fold (211-fold reference and 189-fold variant coverage). (b1) Validating our finding with Sanger sequencing, red arrow indicates the position of the point mutation. The mutation is present in both directions. (a2) Visualizing the alignment of the sequencing reads covering the $A T P 7 B$ c.1707+2dupT heterozygous insertion mutation. The coverage was 399 -fold (188-fold reference and 211-fold variant coverage). (b2) Validating our finding with Sanger-sequencing, red arrow indicates the position of the insertion. The mutation is present in both directions.

accuracy was $99.2 \%$. The average base coverage depth was 1883 (Table 2). The number of identified variants per sample was between 8 and 13; however most of them were known as non-disease-causing variants.

Overall, we found nine disease-causing variants. The most frequent mutation was p.His1069Gln (exon 14, ATP loop) detected in four patients. One novel missense mutation (p.Ala1270Ile, exon 18, ATP hinge vide Figure 1) and three well-known missense mutations (p.Arg969Gln, exon 13, TM6; p.Ala1063Val, exon 14, ATP loop, and p.Leu1305Pro, exon 19 bet ATP hinge/TM7), three frame-shift mutations
(c1707+2dupT, exon 4, Cu6; p.Met769-fs exon 8 TM4, and p.Ala1135-fs exon 15 ATP loop), and one nonsense mutation (p.Gln1351Stop, exon 20, TM8) were detected. All of these variants had been validated by Sanger sequencing.

\section{Discussion}

Although there is an international diagnostic score system for WD [10], the set-up of the diagnosis remains a great challenge in many cases. The signs and symptoms are very colorful, and most of the criteria have relatively low sensitivity and/or 
TABle 2: Per sample and per amplicon coverage data.

\begin{tabular}{|c|c|c|c|c|c|c|c|c|c|c|}
\hline Chromosome & $\begin{array}{c}\text { Amplicon } \\
\text { start }\end{array}$ & $\begin{array}{l}\text { Amplicon } \\
\text { end }\end{array}$ & Amplicon ID & Gene ID & Patient 1 & Patient 2 & Patient 3 & Patient 4 & Patient 5 & Patient 6 \\
\hline chr13 & 52520358 & 52520440 & AMPL1102520485 & ATP7B & 812 & 484 & 378 & 9467 & 3557 & 3275 \\
\hline chr13 & 52542530 & 52542653 & AMPL1102672008 & ATP7B & 951 & 390 & 145 & 3149 & 5510 & 3183 \\
\hline $\operatorname{chr} 13$ & 52538909 & 52539030 & AMPL1404436522 & ATP7B & 9 & 2 & 2 & 2 & 4 & 46 \\
\hline $\operatorname{chr13}$ & 52523735 & 52523838 & AMPL561308261 & ATP7B & 417 & 285 & 89 & 245 & 683 & 2611 \\
\hline $\operatorname{chr13}$ & 52518244 & 52518356 & AMPL561308388 & ATP7B & 1140 & 520 & 339 & 7934 & 6166 & 3595 \\
\hline $\operatorname{chr} 13$ & 52516492 & 52516613 & AMPL561312436 & ATP7B & 600 & 309 & 70 & 1510 & 3218 & 2152 \\
\hline $\operatorname{chr} 13$ & 52515134 & 52515253 & AMPL561312709 & ATP7B & 799 & 287 & 74 & 1340 & 3258 & 2733 \\
\hline $\operatorname{chr13}$ & 52520435 & 52520563 & AMPL561312859 & ATP7B & 622 & 308 & 149 & 409 & 906 & 2130 \\
\hline $\operatorname{chrl3}$ & 52511616 & 52511742 & AMPL561313522 & ATP7B & 343 & 233 & 58 & 1462 & 2168 & 1069 \\
\hline $\operatorname{chr} 13$ & 52548475 & 52548562 & AMPL561315457 & ATP7B & 740 & 557 & 248 & 660 & 1251 & 3869 \\
\hline $\operatorname{chr} 13$ & 52548014 & 52548135 & AMPL561316164 & ATP7B & 506 & 109 & 93 & 244 & 614 & 3077 \\
\hline $\operatorname{chrl3}$ & 52535952 & 52536073 & AMPL561319098 & ATP7B & 345 & 332 & 62 & 1845 & 3919 & 1350 \\
\hline $\operatorname{chr} 13$ & 52532445 & 52532575 & AMPL561319439 & ATP7B & 495 & 382 & 75 & 260 & 477 & 2421 \\
\hline $\operatorname{chr} 13$ & 52518332 & 52518433 & AMPL561320185 & ATP7B & 445 & 574 & 270 & 852 & 1305 & 2147 \\
\hline $\operatorname{chr} 13$ & 52511401 & 52511536 & AMPL561321003 & ATP7B & 899 & 422 & 181 & 3036 & 4216 & 2756 \\
\hline $\operatorname{chr} 13$ & 52523839 & 52523934 & AMPL561322321 & ATP7B & 802 & 389 & 181 & 3872 & 3781 & 2867 \\
\hline $\operatorname{chr13}$ & 52520556 & 52520638 & AMPL561322791 & ATP7B & 836 & 630 & 362 & 9187 & 4465 & 3540 \\
\hline $\operatorname{chr13}$ & 52534277 & 52534394 & AMPL561324803 & ATP7B & 670 & 295 & 126 & 3402 & 3937 & 2458 \\
\hline $\operatorname{chr} 13$ & 52542654 & 52542747 & AMPL561326888 & ATP7B & 755 & 503 & 272 & 811 & 1791 & 3775 \\
\hline $\operatorname{chr13}$ & 52524407 & 52524535 & AMPL561327019 & ATP7B & 215 & 139 & 38 & 126 & 250 & 1023 \\
\hline $\operatorname{chr13}$ & 52516614 & 52516708 & AMPL561328057 & ATP7B & 546 & 401 & 206 & 591 & 1259 & 2779 \\
\hline $\operatorname{chr} 13$ & 52515254 & 52515365 & AMPL561328062 & ATP7B & 768 & 466 & 191 & 508 & 1239 & 3144 \\
\hline $\operatorname{chr13}$ & 52513223 & 52513345 & AMPL561328524 & ATP7B & 454 & 382 & 113 & 239 & 732 & 1771 \\
\hline $\operatorname{chr13}$ & 52548563 & 52548672 & AMPL561329883 & ATP7B & 836 & 384 & 149 & 3334 & 4618 & 3056 \\
\hline $\operatorname{chr} 13$ & 52511743 & 52511824 & AMPL561330934 & ATP7B & 718 & 556 & 323 & 946 & 1461 & 3040 \\
\hline $\operatorname{chr13}$ & 52532576 & 52532683 & AMPL561335846 & ATP7B & 671 & 433 & 227 & 5882 & 4370 & 2749 \\
\hline $\operatorname{chrl3}$ & 52511497 & 52511615 & AMPL561335849 & ATP7B & 813 & 494 & 353 & 857 & 1761 & 3297 \\
\hline $\operatorname{chr} 13$ & 52549016 & 52549114 & AMPL561337443 & ATP7B & 453 & 347 & 167 & 3818 & 3121 & 2050 \\
\hline $\operatorname{chr} 13$ & 52534395 & 52534476 & AMPL561338245 & ATP7B & 734 & 542 & 476 & 1282 & 2378 & 2732 \\
\hline $\operatorname{chr13}$ & 52539048 & 52539119 & AMPL561339230 & ATP7B & 532 & 448 & 394 & 9393 & 3563 & 2527 \\
\hline $\operatorname{chr} 13$ & 52544567 & 52544690 & AMPL561342268 & ATP7B & 806 & 449 & 130 & 2523 & 3519 & 2674 \\
\hline $\operatorname{chr} 13$ & 52548673 & 52548782 & AMPL561343055 & ATP7B & 512 & 446 & 158 & 513 & 1052 & 3022 \\
\hline $\operatorname{chr} 13$ & 52548136 & 52548260 & AMPL561345064 & ATP7B & 826 & 442 & 128 & 2692 & 4578 & 2624 \\
\hline $\operatorname{chr13}$ & 52549115 & 52549227 & AMPL561347059 & ATP7B & 469 & 369 & 144 & 398 & 771 & 2612 \\
\hline $\operatorname{chr} 13$ & 52539120 & 52539203 & AMPL561347740 & ATP7B & 787 & 737 & 877 & 3031 & 2553 & 4243 \\
\hline $\operatorname{chr13}$ & 52509711 & 52509847 & AMPL561353128 & ATP7B & 329 & 280 & 70 & 208 & 669 & 2063 \\
\hline $\operatorname{chr13}$ & 52549228 & 52549346 & AMPL561354995 & ATP7B & 720 & 232 & 59 & 900 & 2717 & 2418 \\
\hline $\operatorname{chr} 13$ & 52508853 & 52508964 & AMPL561358361 & ATP7B & 660 & 455 & 181 & 4930 & 3832 & 2325 \\
\hline $\operatorname{chr13}$ & 52548783 & 52548894 & AMPL561361353 & ATP7B & 1286 & 639 & 267 & 7815 & 7277 & 3588 \\
\hline $\operatorname{chr13}$ & 52544691 & 52544813 & AMPL561365512 & ATP7B & 542 & 354 & 150 & 336 & 898 & 2919 \\
\hline $\operatorname{chr} 13$ & 52508959 & 52509084 & AMPL561366430 & ATP7B & 694 & 574 & 217 & 630 & 1344 & 2208 \\
\hline $\operatorname{chr} 13$ & 52524093 & 52524178 & AMPL561367088 & ATP7B & 368 & 315 & 5 & 31 & 82 & 2778 \\
\hline $\operatorname{chr13}$ & 52524179 & 52524298 & AMPL561373391 & ATP7B & 725 & 390 & 108 & 2697 & 4100 & 2724 \\
\hline $\operatorname{chrl3}$ & 52544814 & 52544931 & AMPL561373418 & ATP7B & 235 & 52 & 34 & 618 & 1093 & 1651 \\
\hline $\operatorname{chr} 13$ & 52548893 & 52549018 & AMPL561375011 & ATP7B & 498 & 471 & 103 & 239 & 550 & 2166 \\
\hline $\operatorname{chr} 13$ & 52509084 & 52509181 & AMPL561375394 & ATP7B & 719 & 461 & 144 & 5037 & 3879 & 2163 \\
\hline $\operatorname{chr13}$ & 52531644 & 52531756 & AMPL561379526 & ATP7B & 512 & 234 & 80 & 1601 & 2656 & 2155 \\
\hline $\operatorname{chr} 13$ & 52548255 & 52548381 & AMPL561399016 & ATP7B & 467 & 446 & 98 & 286 & 676 & 2965 \\
\hline $\operatorname{chr13}$ & 52548382 & 52548474 & AMPL561401395 & ATP7B & 247 & 124 & 69 & 1000 & 357 & 2414 \\
\hline chrl3 & 52513106 & 52513229 & AMPL561308165 & $\begin{array}{c}\text { c. } 3699+27 \mathrm{~T}>\mathrm{C}, \\
\text { ATP7B }\end{array}$ & 560 & 372 & 88 & 1778 & 3799 & 2176 \\
\hline chrl3 & 52585387 & 52585514 & AMPL561308108 & $\begin{array}{c}\text { c. }-36 \mathrm{C}>\mathrm{T}, \text { c. }-75 \mathrm{~A}>\mathrm{C} \text {, } \\
\text { ATP7B }\end{array}$ & 294 & 287 & 110 & 313 & 616 & 2859 \\
\hline chrl3 & 52585831 & 52585931 & AMPL1275480480 & ATP7B & 493 & 397 & 129 & 807 & 780 & 1389 \\
\hline $\operatorname{chrl3}$ & 52585851 & 52585971 & AMPL1275480698 & ATP7B & 458 & 140 & 55 & 1205 & 1642 & 811 \\
\hline chrl3 & 52534093 & 52534223 & AMPL1275484758 & ATP7B & 140 & 230 & 35 & 69 & 187 & 2149 \\
\hline chr13 & 52585478 & 52585613 & AMPL561317674 & ATP7B & 560 & 186 & 58 & 997 & 1619 & 1979 \\
\hline
\end{tabular}


specificity. Although genetic testing in itself can ascertain the diagnosis, it is limited by the great variety of the mutations. It is also difficult to screen the siblings of a WD patient, especially of those who do not have identified mutations, since the abnormal laboratory results of copper metabolism may occur in heterozygous carriers. The tight observation of these siblings and the doubt if they are affected can make their life very stressful and uncomfortable. The detection of the mutations in the index patient and searching for the same in the siblings can resolve this problem.

The whole gene analysis of $A T P 7 B$ by PCR and capillary sequencing in a large cohort of WD patients has been recently published [11]. According to our knowledge based on PubMed data this is the first report on next-generation sequencing of the $A T P 7 B$ gene for genetic diagnosis of Wilson's disease in a clinical setting. Since the disease-causing mutations may occur in the whole length of the gene and every exon could be affected, the genetic examination by classical methods is ponderous and time-consuming.

Our study clearly shows the great benefit of NGS. The compound heterozygosity has been proved in each patient within a very short examination time. Previously, we published that p.His1069Gln mutation is most common one in Hungary (71\%) similar to other Central and Eastern European countries [6,12-14]. Results of this study are in concordance with the former epidemiological data, since this mutation was confirmed in the majority of the cases, in 4 out of 6 . Among the eight other mutations we found, there is one novel mutation in exon 18 which is a missense mutation causing an asparagine-isoleucine change in the transporter. Interestingly, the mutations beyond p.His1069Gln occurred only in one allele of a single patient.

However, it is already well known that p.His1069Gln homozygous mutation tends to relate with neurological symptoms; the effect of other infrequent mutations on the phenotype is hard to be examined due to the low number of cases (vide Table 1) [15].

p.Ala1063Val mutation detected in one patient who has been diagnosed with WD prior to genetic testing is thought to be a non-disease-causing variant according to the Wilson Disease Mutation Database, although only one publication suggested that it might be a polymorphism [4]. On the other hand, subsequent data show that it might be a variant of unknown significance (VUS) [16]. Furthermore it was the one and only nucleotide change in a WD family analyzed by Loudianos et al. [17]. Overall it seems that p.Ala1063Val mutation still might be associated with Wilson's disease.

NGS gave a tremendous benefit for a 47-year-old patient with acute on chronic liver failure. Although nearly all patients with ALF due to Wilson's disease are potentially diagnosed (or suspicion is very high) with use of simple biochemical and laboratory criteria (ratio of alkaline phosphatase to bilirubin, ratio of AST to ALT, and Coombs negative hemolytic anemia) [18], the diagnosis may require an urgent genetic testing of all mutations. In some patients the laboratory data alone cannot give enough scores in the international score system [10], which is required by Eurotransplant program for donor liver allocation. In our case, the results of D-penicillamine test and the NGS arrived simultaneously, confirming Wilson's disease. According to the actual regulation of Eurotransplant Organization in case of acute on chronic liver failure only WD and Budd-Chiari syndrome are accepted as indication for urgent transplantation. Identifying mutations in both alleles gave a clear-cut evidence of the disease despite lack of Kayser-Fleischer ring, lack of neurological symptoms, and p.His1069Gln mutation. Thanks to the quick diagnosis the patient has been transplanted within two days and survived, and he is still in good condition one year later.

\section{Conclusion}

According to our results we found next-generation sequencing to be a very useful, reliable, time-saving, and cost effective method for diagnosing Wilson's disease in selected cases.

\section{Conflict of Interests}

The authors declare that there is no conflict of interests regarding the publication of this paper.

\section{Authors' Contribution}

Dániel Németh and Kristóf Árvai have made an equal contribution.

\section{References}

[1] K. Petrukhin, S. G. Fischer, M. Pirastu et al., "Mapping, cloning and genetic characterization of the region containing the Wilson disease gene," Nature Genetics, vol. 5, no. 4, pp. 338343, 1993.

[2] P. Ferenci, "Regional distribution of mutations of the ATP7B gene in patients with Wilson disease: impact on genetic testing," Human Genetics, vol. 120, no. 2, pp. 151-159, 2006.

[3] G. Loudianos, V. Dessi, M. Lovicu et al., "Molecular characterization of Wilson disease in the Sardinian population-evidence of a founder effect," Human Mutation, vol. 14, no. 4, pp. 294-303, 1999.

[4] M. S. Nanji, V. T. T. Nguyen, J. H. Kawasoe et al., "Haplotype and mutation analysis in Japanese patients with Wilson disease," American Journal of Human Genetics, vol. 60, no. 6, pp. 14231429, 1997.

[5] E. K. Kim, O. J. Yoo, K. Y. Song et al., "Identification of three novel mutations and a high frequency of the Arg778Leu mutation in Korean patients with Wilson disease," Human Mutation, vol. 11, no. 4, pp. 275-278, 1998.

[6] A. Folhoffer, P. Ferenci, T. Csak et al., "Novel mutations of the ATP7B gene among 109 Hungarian patients with Wilson's disease," European Journal of Gastroenterology and Hepatology, vol. 19, no. 2, pp. 105-111, 2007.

[7] J. M. Walshe, "Diagnosis and treatment of presymptomatic Wilson's disease," The Lancet, vol. 332, no. 8608, pp. 435-437, 1988.

[8] A. von Bubnoff, "Next-generation sequencing: the race is on," Cell, vol. 132, no. 5, pp. 721-723, 2008.

[9] B. Balla, K. Árvai, P. Horváth et al., "Fast and robust nextgeneration sequencing technique using ion torrent personal 
genome machine for the screening of neurofibromatosis type 1 (NF1) gene," Journal of Molecular Neuroscience, vol. 53, no. 2, pp. 204-210, 2014.

[10] P. Ferenci, K. Caca, G. Loudianos et al., "Diagnosis and phenotypic classification of Wilson disease," Liver International, vol. 23, no. 3, pp. 139-142, 2003.

[11] A. J. Coffey, M. Durkie, S. Hague et al., "A genetic study of Wilson's disease in the United Kingdom," Brain, vol. 136, no. 5, pp. 1476-1487, 2013.

[12] B. Tarnacka, G. Gromadzka, M. Rodo, P. Mierzejewski, and A. Czlonkowska, "Frequency of His1069Gln and Gly1267Lys mutations in Polish Wilson's disease population," European Journal of Neurology, vol. 7, no. 5, pp. 495-498, 2000.

[13] K. Caca, P. Ferenci, H. J. Kühn et al., "High prevalence of the H1069Q mutation in east german patients with wilson disease: rapid detection of mutations by limited sequencing and phenotype-genotype analysis," Journal of Hepatology, vol. 35, no. 5, pp. 575-581, 2001.

[14] S. Vrabelova, O. Letocha, M. Borsky, and L. Kozak, "Mutation analysis of the ATP7B gene and genotype/phenotype correlation in 227 patients with Wilson disease," Molecular Genetics and Metabolism, vol. 86, no. 1-2, pp. 277-285, 2005.

[15] P. Ferenci, "Phenotype-genotype correlations in patients with Wilson's disease," Annals of the New York Academy of Sciences, vol. 1315, no. 1, pp. 1-5, 2014.

[16] D. Curtis, M. Durkie, P. Balac (Morris) et al., "A study of Wilson disease mutations in Britain," Human Mutation, vol. 14, no. 4, pp. 304-311, 1999.

[17] G. Loudianos, V. Dessi, M. Lovicu et al., "Mutation analysis in patients of Mediterranean descent with Wilson disease: identification of 19 novel mutations," Journal of Medical Genetics, vol. 36, no. 11, pp. 833-836, 1999.

[18] J. D. Korman, I. Volenberg, J. Balko et al., "Screening for Wilson disease in acute liver failure: a comparison of currently available diagnostic tests," Hepatology, vol. 48, no. 4, pp. 1167-1174, 2008. 


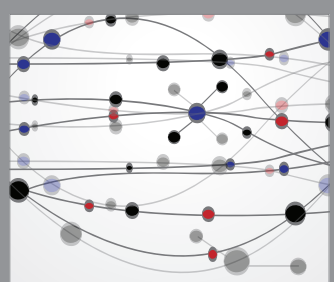

The Scientific World Journal
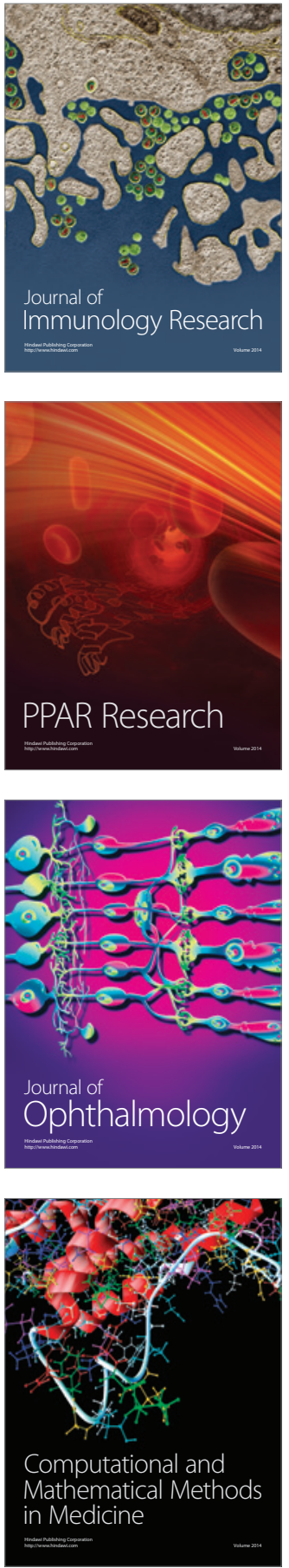

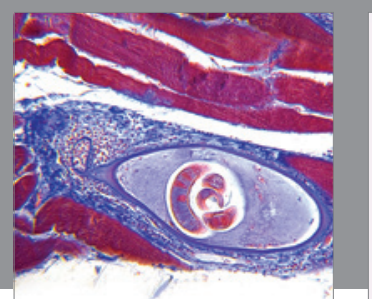

Gastroenterology Research and Practice

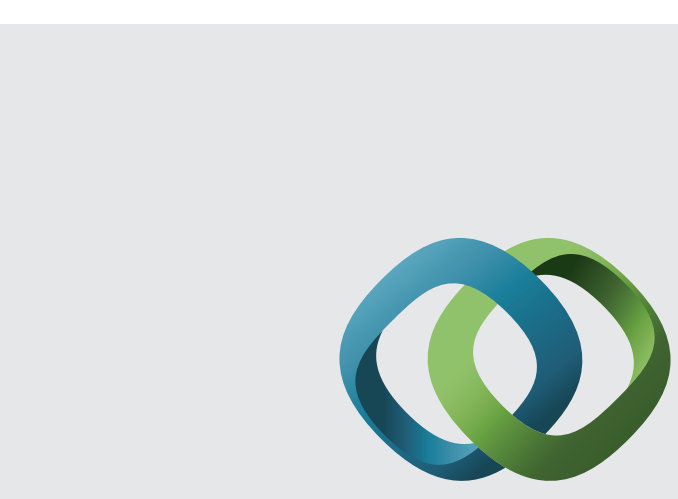

\section{Hindawi}

Submit your manuscripts at

http://www.hindawi.com
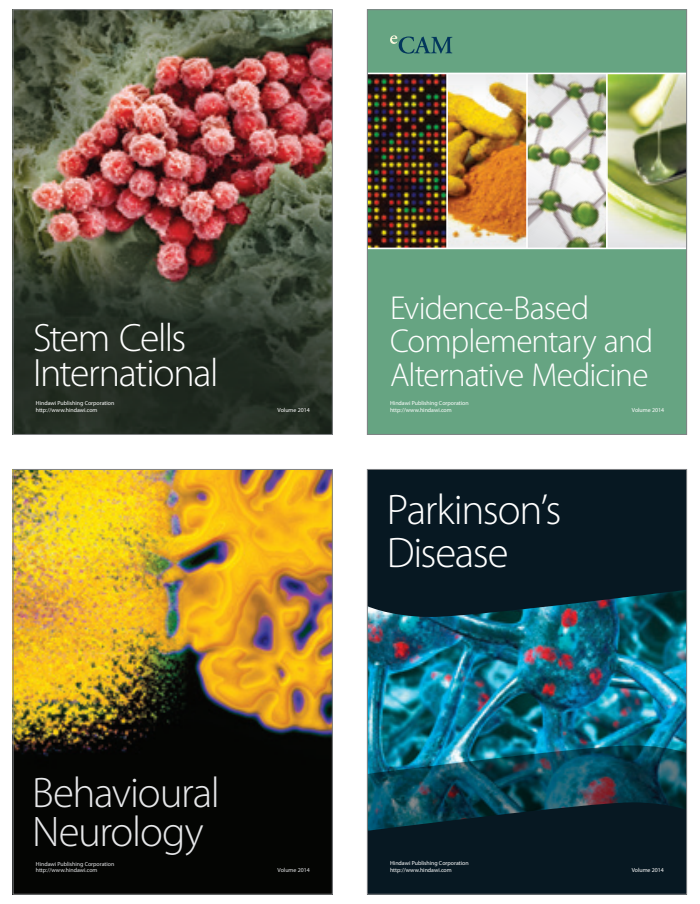
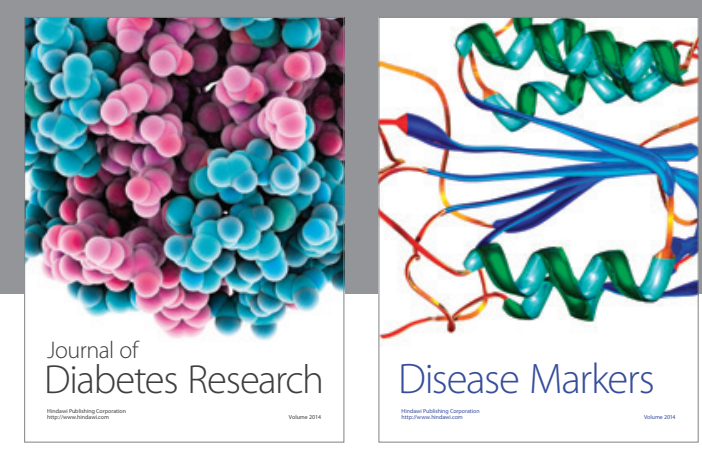

Disease Markers
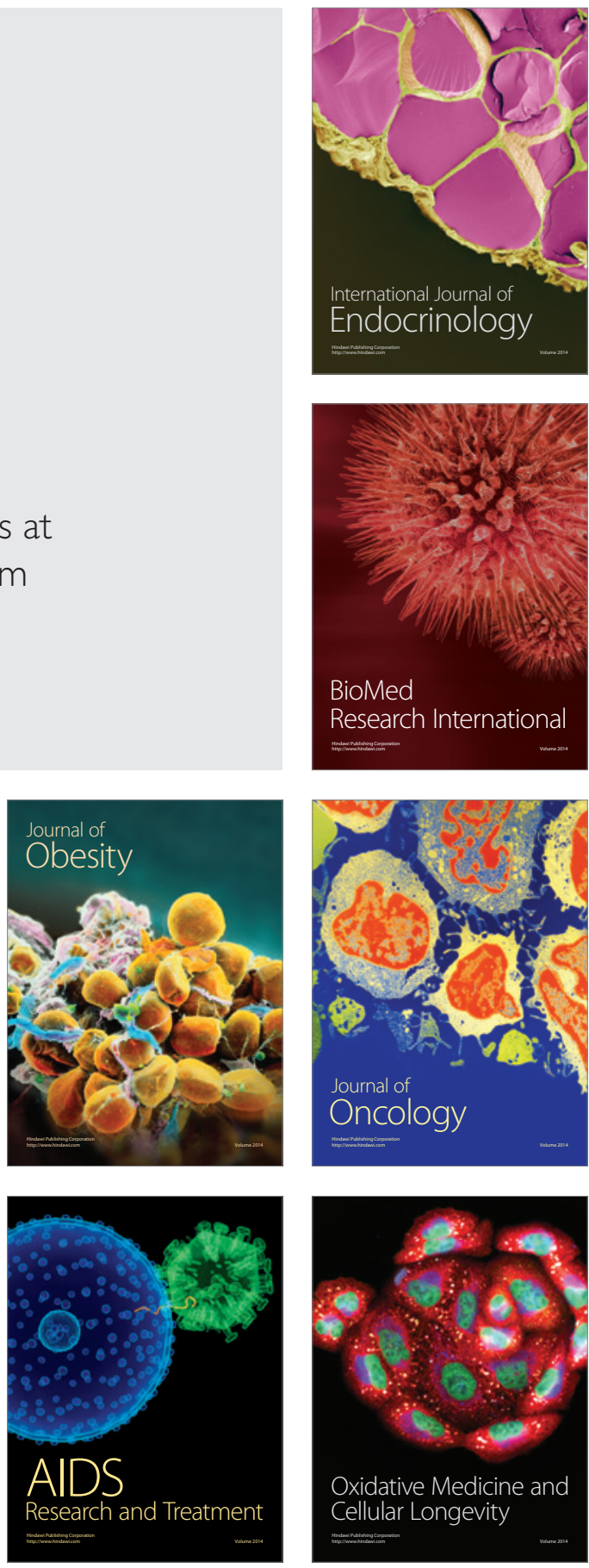